\title{
Anatomically Informed Basis Functions
}

\author{
Stefan J . Kiebel,* Rainer Goebel, $†$ and Karl J . Fristonł \\ *Department of Neurology, Friedrich-Schiller-University, 07740 J ena, Germany; †Max-Planck-Institut for Brain Research, Deutschordenstr. 46, \\ 60528 Frankfurt/ M., Germany; łThe Wellcome Department of Cognitive Neurology, The Institute of Neurol ogy, London, United Kingdom
}

Received September 16, 1999

This paper introduces the general framework, concepts, and procedures of anatomically informed basis functions (AIBF), a new method for the analysis of functional magnetic resonance imaging ( $\mathrm{FMRI}$ ) data. In contradistinction to existing voxel-based univariate or multivariate methods the approach described here can incorporate various forms of prior anatomical knowledge to specify sophisticated spatiotemporal models for fMRI time-series. In particular, we focus on anatomical prior knowledge, based on reconstructed gray matter surfaces and assumptions about the location and spatial smoothness of the blood oxygenation level dependent (BOLD) effect. After reconstruction of the grey matter surface from an individual's high-resolution T1-weighted MRI, we specify a set of anatomically informed basis functions, fit the model parameters for a single time point, using a regularized solution, and finally make inferences about the estimated parameters over time. Significant effects, induced by the experimental paradigm, can then be visualized in the native voxel-space or on the reconstructed folded, inflated, or flattened cortical surface. As an example, we apply the approach to a fMRI study (finger opposition task) and compare the results to those of a voxel-based analysis as implemented in the Statistical Parametric Mapping package (SPM99). Additionally, we show, using simulated data, that the approach offers several desirable features particularly in terms of superresolution and localization.

2000 Academic Press

Key Words: fMRI; computational neuroanatomy; modelling; spatiotemporal model; statistical inference.

\section{INTRODUCTION}

In functional brain imaging, the analysis of $\mathrm{FMRI}$ data has become an increasingly important issue. The underlying task is to characterize the functional data observed in space and time in terms of some interpretable and reduced representation of the experimentally induced effects. Many methods have been proposed that can be divided into univariate and multivariate approaches.
With a univariate approach, one typically uses prior knowledge about experimentally evoked signals over time to project the time-series, at any voxel position, into a low-dimensional parameter space (typically defined by a design matrix). Theremaining task is then to assess the spatial autocorrelations and make inferences about the observations in voxel-space. The overwhelming proportion of methods published belong to this class. In contrast, multivariate approaches can identify a few spatiotemporal components that summarize the observed data without reference to any predictions. Though the latter approach appears to be more comprehensive, because it is explicitly spatiotemporal (e.g., eigenimage analysis), it is often rather difficult to interpret the spatiotemporal components that ensue, largely because this sort of dataled characterization does not embody prior information about interesting temporal responses. Although design matrices can be used in multivariate analyses (e.g., canonical variates analysis), these analyses are compromised by the sample-size (number of scans) to dimensionality (number of voxels) ratio.

Mass-univariate, voxel-based approaches have an advantage over multivariate data-led approaches, because the temporal projection space (design matrix) is defined explicitly by the researcher. Conventional analyses of this sort start by convolving the data with a lowpass spatial filter, which (i) renders the point spread function (PSF) approximately stationary and (ii) enhances sensitivity to underlying (extended) physiological signal sources by virtue of the matched filter theorem. However, the resolution of the resulting statistical maps is compromised. Furthermore, convolution with a lowpass filter implicitly implements a spatial model of the inherent spatial smoothness of the BOLD effect. If one assumes that the source of underlying signal is restricted to the gray matter, it is obvious that the spatial model implied by a spherical lowpass filter is inappropriate: It does not incorporate knowledge about the local anatomical distribution of potential signal sources in the folded cortical sheet.

Anatomically informed basis functions (AIBF), presented in this work, define a new class of models, which 
allow the incorporation of prior neuroanatomical knowledge provided that this information can be represented as a set of basis functions. As an example, we describe an anatomical model based on an individual's reconstructed cortical surface and show that spatial resolution and sensitivity for extended cortical sources is improved as compared to a conventional voxel-wise analysis. We thereby implicitly introduce a general framework for including other types of prior anatomical knowledge.

We applied the method to fMRI measurements (finger opposition task) of five subjects and to simulated data, where the exact locations and time courses of the underlying signals were known. We show, by comparison with a voxel-based approach (SPM99), that the method enhances resolution and interpretability of the resulting statistical maps.

\section{ANATOMICALLY INFORMED BASIS FUNCTIONS}

\section{Overview}

The problem addressed by anatomically informed basis functions can bestated as follows: Given the assumptions that (i) the BOLD effects are located somewhere on the cortical surface and (ii) can be characterized by a spatially smooth distribution on that surface, what is the spatial distribution of the BOLD signal, which best explains the functional observations?

If both assumptions are valid, we can describe an anatomically informed model that enables us to characterize any measured functional data directly on the cortical surface. As we will show later there are three major advantages of the technique when compared to voxel-based methods: (i) The method does not use any additional three-dimensional spatial lowpass filtering, but operates directly on the realigned unsmoothed data. (ii) The method provides superresolution and (iii) by virtue of the partially distinct spatial characteristics of underlying effects, AIBF can be used to further differentiate among underlying signal sources.

AIBF can also be used to implement an anatomically constrained least-squares deconvolution of the PSF for low-resolution data to remove PSF effects. This applies when the intrinsic PSF is large relative to voxel size. However, we do not deal with this application in this paper, because we restrict ourselves to fMRI analyses.

In the following, we describe the approach in three subsections. First, we will specify the spatial model that embodies anatomical a priori knowledge about a functional observation. In the second subsection, the anatomical model is fitted to a series of functional volumes observed over time, to generate a spatiotemporal parameter matrix. Finally, we deal with inference about the effects in the time domain. In this paper, we are assuming that the spatiotemporal basis set can be factorized into a spatial and temporal basis set, where the latter corresponds to conventional re- gressors in a design matrix. This allows us to first estimate the spatial distribution of sources at each time point and then estimate, and make inferences about, their expression over time.

\section{The Spatial Mode}

Surfacereconstruction. A necessary prerequisite to the specification of a set of surface-based basis functions is the reconstruction of cortical surfaces. These reconstructions were obtained with the software package BrainVoyager (Version 3.5, Brain Innovations, Rainer Goebel). For each subject, a T1-weighted anatomical image was acquired on a clinical 1.5 T Siemens Vision unit (Siemens $\mathrm{GmbH}$, E rlangen) with TR $=15$ $\mathrm{ms}, \mathrm{TE}=5 \mathrm{~ms}$, flip angle $=30^{\circ}$. Each image matrix consisted of $256 \times 256$ voxels, 192 partitions, sagittal orientation, voxel size $1 \times 1 \times 1 \mathrm{~mm}^{3}$.

The process of surface reconstruction, inflation, and flattening as implemented in BrainVoyager is described elsewhere (Linden et al., 1999). We focus here on the properties of the various surfaces generated.

To reconstruct the individual cortical surface, we segment the cerebral white matter, the ventricles, and deep gray matter structures like the putamen and thalamus of both hemispheres. The segmented voxel-based partition of each hemisphere is transformed to a vertex-based surface $S_{0}=\left(V_{0}, F\right)$ by triangularization of the outside voxel faces, where $\mathrm{V}_{0}$ is a $\mathrm{N}_{\mathrm{v}_{0}} \times 3$-matrix of vertex coordinates, $\mathrm{F}$ is a $\mathrm{N}_{\mathrm{F}} \times 3$-matrix of vertex indices, $\mathrm{N}_{\mathrm{V}_{0}}$ and $\mathrm{N}_{\mathrm{F}}$ are the number of vertices and faces. Since the surface $S_{0}$, lying between white and gray matter partitions, reflects the coarse voxel-based discretized approximation to the (real) underlying surface, which is assumed to be spatially smooth (i.e., local Gaussian curvature values are bound by some maximum value), the coordinates described by $V_{0}$ are spatially smoothed, with respect to the local vertex neighbourhood. $\mathrm{V}_{0}$ is thus transformed to a smooth representation of the white matter surface $S_{w}=\left(V_{w}, F\right)$. In the next step, a surface lying within the gray matter sheet is identified by translating the vertices in $V_{w}$ using a local iterative intensity-based optimization routine. This gives a representation of the underlying gray matter surface $S_{G}=\left(V_{G}, F\right)$ (Fig. 1a). We then compute an inflated surface $S_{1}=\left(V_{1}, F\right)$ (Fig. 1b) of the approximation to the cortical sheet $\mathrm{S}_{\mathrm{G}}$. After placing several cuts on the medial side of the hemisphere, we project the inflated brain surface onto a plane and reduce distortions that arise from the projection and intrinsic curvature of the cortical surface. The result is a flattened map (Fig. 1C) of one cortical hemisphere $\mathrm{S}_{\mathrm{F}}=\left(\mathrm{V}_{\mathrm{F}}, \mathrm{F}\right)$.

N ote that in all surface representations the face matrix $F$ never changes, only the vertex coordinates matrices $V_{W}, V_{G}, V_{1}$, and $V_{F}$ are modified to describe different surfaces. In this way, the coordinate 

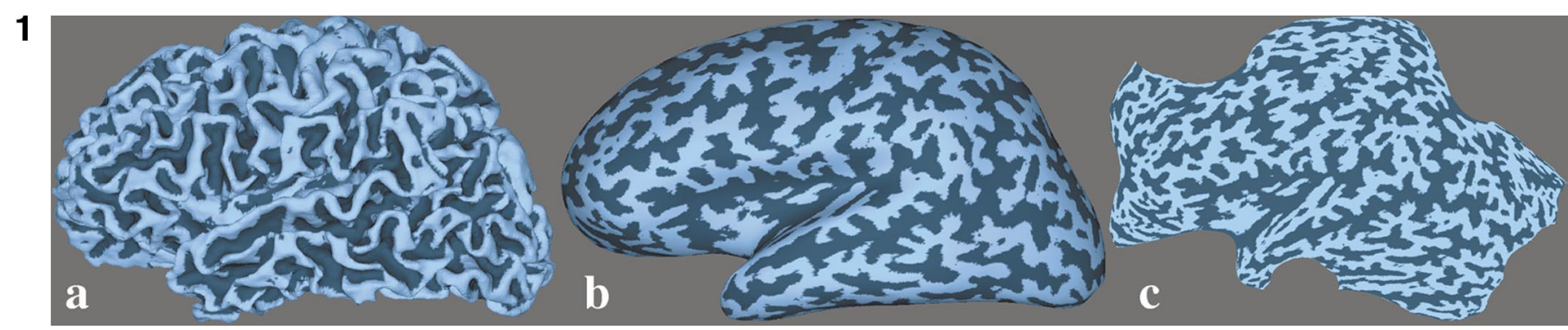

\section{2}
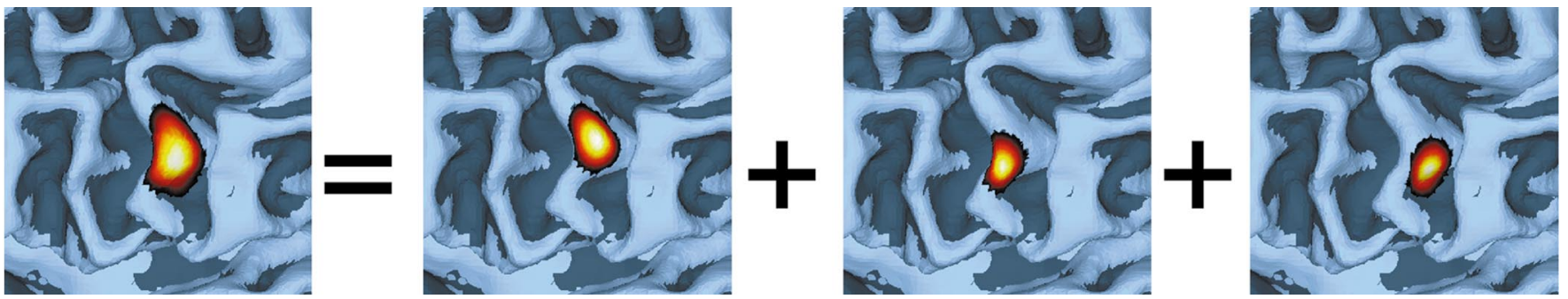

3
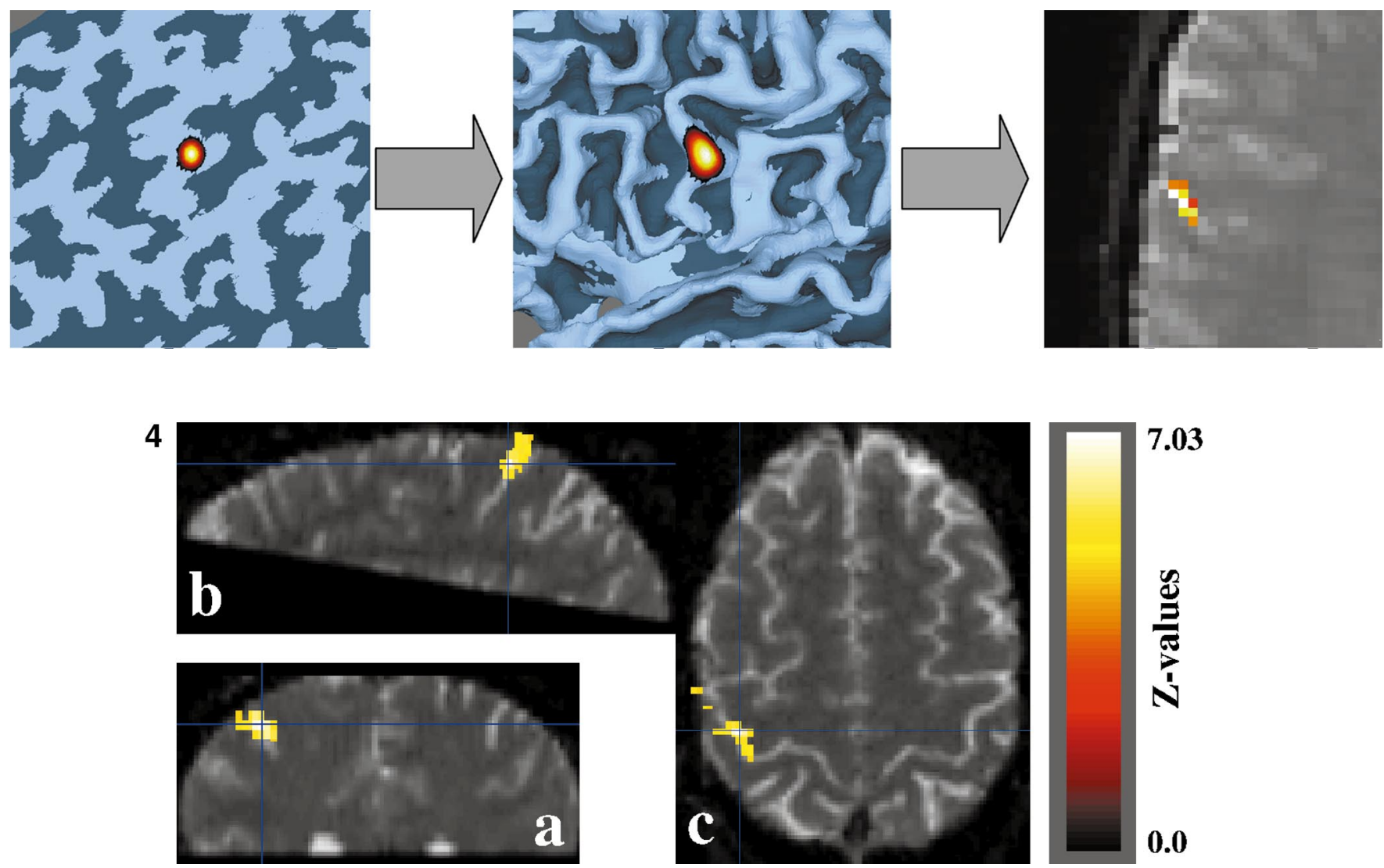

FIG. 1. Cortical surface of the left hemisphere in three different representations: (a) folded, (b) inflated, (c) flattened.

FIG. 2. Concept of smooth overlapping spatial basis functions: The distribution on the left can be expressed as a linear combination of the three basis functions on the right.

FIG. 3. Different representations of a basis function. (Left) Two-dimensional Gaussian kernel with $1 \mathrm{~mm}$ width in $\mathrm{x}$ - and y-direction. (Middle) The same basis function transformed to the original reconstructed cortical surface. (Right) A transverse slice of its representation in voxel-space.

FIG. 4. Results of a voxel-based analysis (SPM99) of a single subject (thumb versus middle finger tapping task). Maximum peak displayed on a (a) coronal, (b) sagittal, and (c) transverse slice. 
transformation between two surfaces is implemented by an exchange of vertex coordinates matrices.

Specification of spatial model. Weassume that the individual gray matter surface $S_{G}=\left(V_{G}, F\right)$ is a good approximation to the real underlying gray matter surface in the individual's anatomical space. Given a functional observation vector $Y$ in voxel-space, our aim is to estimate a smooth distribution $f$ on the vertices $V_{G}$ of the reconstructed gray matter surface that best (in some sense) explains the observed Y, i.e.,

$$
\mathrm{Y}=\mathrm{g}\left(\mathrm{f}\left(\mathrm{V}_{\mathrm{G}}\right)\right)+\epsilon,
$$

where $\epsilon$ is the residual component and $g$ is an operator (to be described later), which transforms intensities defined in vertex-space to the voxel-space of Y. Typically, one reconstructed hemisphere consists of roughly 130,000 vertices, whereas the number of observed gray matter voxels in one hemisphere (e.g., 20 partitions, $128 \times 128$ matrices, voxel size $1.8 \times 1.8 \times 3 \mathrm{~mm}^{3}$ ) is much less, roughly 12,000; i.e., the problem is underdetermined. One key to solve this problem is the assumption about the smoothness of $\mathrm{f}$. A smooth distribution can be modelled by less parameters $N_{p}$ than the number of vertices $\mathrm{N}_{\mathrm{v}_{\mathrm{G}}}$ so the idea is to approximate $f$ by a model that is determined by a small set of parameters. We choose to model the distribution $f$ with a linear combination of local, smooth, and partially overlapping spatial basis functions defined on $\mathrm{V}_{\mathrm{G}}$. Figure 2 illustrates the concept behind this approach.

A necessary prerequisite for the specification of spatial basis functions on the surface $S_{G}$ is the existence of a two-dimensional coordinate system, but due to the folded nature of the gray matter surface, there is no simple way to define such a coordinate system. However, the cortical flat map $S_{F}=\left(V_{F}, F\right)$ is the result of a coordinate transformation from the vertex coordinates $V_{G}$ to a plane; i.e., for any vertex coordinate $v_{G}^{i}$ on $\mathrm{S}_{\mathrm{G}}$ its associated location on $\mathrm{S}_{\mathrm{F}}$ is stored in $\mathrm{V}_{\mathrm{F}}^{\mathrm{i}}$. Since there is a two-dimensional coordinate system available on the cortical flat map $S_{F}$, we define the basis functions on $S_{F}$ and reproject them onto the cortical sheet $S_{G}$ by a coordinate exchange.

Specification of basis functions. There are three conditions the basis set must conform to: (i) Each basis function must cover a small patch of $S_{F}$, (ii) the linear combination of basis functions is able to model smooth distributions on $S_{F}$, and (iii) the approximation of $f$ at any vertex location is implemented as sparsely as possible. Any form of two-dimensional Gaussian, sincfunction, or similar lowpass-kernel functions fulfill the first two criteria, provided that each basis function overlaps with its neighbors to a certain degree. The third condition means that this overlap is not unnecessarily large.

In this work, we use circular Gaussian basis functions in a hexagonal configuration such that the center of each basis function has a fixed (user-specified) distance to the center of its six neighbors. Given a twodimensional coordinate system on the cortical flat map $S_{F}$, the basis function $b_{F}^{j}$ with its center at coordinates $\left(x_{j}, y_{j}\right)$ is defined by

$$
b_{F}^{j}(x, y)=c_{1} \exp \left(\frac{-\left(\left(x-x_{j}\right)^{2}+\left(y-y_{j}\right)^{2}\right.}{2 w^{2}}\right),
$$

where $c_{1}$ is a constant and $\mathrm{w}$ is the (user-specified) width of the Gaussian basis function in the $x$ - and $y$-directions. The hexagonal pattern of the centers of the basis functions are defined by induction; i.e., given that $\left(x_{j}, y_{j}\right)$ is the center of $b_{F}^{j}$, then the centers of its six-neighbor basis functions are $\left(x+d / 2, y+d_{0}\right)$, $(x+d, y), \quad\left(x+d / 2, y-d_{0}\right), \quad\left(x-d / 2, y-d_{0}\right)$, $(x-d, y),\left(x-d / 2, y+d_{o}\right)$, where $d$ is the fixed distance between centers and $d_{o}=\sin \left(60^{\circ}\right) d$. The position of the first basis function is chosen arbitrarily. A basis function is defined only if its center is enclosed by a face of the cortical flat map $S_{F}$.

After specification of the basis functions on the cortical flat map $b_{F}^{j}, j=1, \ldots, N_{p}$, where $N_{p}$ is the number of basis functions, each $b_{F}^{j}$ is reprojected onto the original folded surface $S_{G}$ by a vertex coordinate exchange between $V_{F}$ and $V_{G}$ to give folded basis functions $b_{G}^{j}$. Since the $b_{G}^{j}$ are still in vertex-space, the final step is to embed each basis function $b_{G}^{j}$ into the voxel-space. To do this, we define an operator $\mathrm{g}$ (Eq. (1)), which integrates over each voxel $k$ the surface of a given basis function $b_{G}^{j}$ multiplied by the height of the basis function; i.e., g returns the integral of each basis function in folded vertex-space encompassed by the voxel. This transforms the basis functions $b_{G}^{j}$ in vertex-space to $b_{Y}^{j}\left(b_{Y}^{j}=b_{Y_{1}}^{j}, \ldots, b_{Y_{N_{K}}}^{j}\right)$ in voxel-space, where $N_{K}$ is the number of functional voxels. This basis set is now used to model, in a linear combination, the functional observation $Y$.

In Fig. 3, we show the process of transforming a basis function from its initial representation on the cortical flat map to its intermediate state on the folded cortical map and to its final representation in voxel-space.

Note that one could apply an additional convolution $\mathrm{L}$ to the basis functions in voxel-space $b_{Y}^{j}$, if the width of the image PSF is larger than the voxel size. This is not necessary for $\mathrm{FMRI}$, since here we assume that $\mathrm{L}$ $=\mathrm{I}$ (in Toeplitz notation, where I is the identity matrix). For example with PET (positron emission tomography) data, one could model the positron travel by convolution of $b_{Y}^{j}$ with an isotropic lowpass filter kernel and the effects of the measurement process itself by an additional convolution with an anisotropic lowpass filter kernel. This particular extension will be dealt with in a separate communication.

Estimating the spatial distribution of activity for one time point. Let $A$ be $\mathrm{N}_{\mathrm{K}} \times \mathrm{N}_{\mathrm{p}}$-matrix, where column 
j of $A$ is the basis function $b_{Y}^{j}$ in voxel-space. Generally, $A=L\left[b_{Y}^{1}|\ldots| l \mid b_{Y}^{N_{p}}\right]$, but for $f M R I$ we set $L=I$ and omit $L$ in the following equations. Then, the model (compare to Eq. (1)) is

$$
\mathrm{Y}=\mathrm{A} \beta+\epsilon,
$$

where $\mathrm{Y}$ is the $\mathrm{N}_{\mathrm{K}}$-dimensional observation vector in voxel-space, $\beta$ is a $N_{p}$-dimensional parameter vector and $\epsilon$ is a $N_{K}$-dimensional residual error vector.

The unknown parameter vector $\beta$ is assessed by a least-squares estimate with zeroth-order regularization (Press et al., 1992), which yields

$$
\hat{\beta}=\left(A^{\top} A+\lambda I\right)^{-1} A^{\top} Y,
$$

where $\lambda$ is a regularization factor and I is the identity matrix of dimension $\mathrm{N}_{\mathrm{p}}$. The zeroth-order regularization tends to minimize the overall length of the vector $\hat{\beta}$, which is necessary if the distance d between centers of neighboring basis functions is large compared to their width w, i.e., adjacent basis functions overlap extensively and are close to collinearity.

The choice for the regularization factor $\lambda$ depends on the spatial overlap and therefore their degree of nonorthogonality. A standard choice for $\lambda$ (Press et al., 1992) is

$$
\lambda=\frac{\operatorname{trace}\left(A^{\top} A\right)}{N_{p}}
$$

and yields acceptable results in our case. In the extreme that adjacent basis functions do not have any overlap or only a small overlap, the regularization factor can be set to zero and $\mathrm{Eq}$. (4) reduces to a standard least-squares solution

$$
\hat{\beta}=\left(A^{\top} A\right)^{-1} A^{\top} Y .
$$

\section{The Temporal Domain}

Given a series of functional observations $Y_{1}, \ldots$,

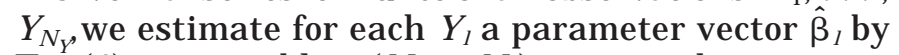
$\mathrm{Eq}$. (4) to assemble a $\left(\mathrm{N}_{\mathrm{Y}} \times \mathrm{N}_{\mathrm{p}}\right)$ estimated parameter matrix

$$
\mathrm{B}^{\top}=\left[\hat{\beta}_{1}|\ldots| \hat{\beta}_{\mathrm{N}_{\mathrm{Y}}}\right]=\left(\mathrm{A}^{\mathrm{T}} \mathrm{A}+\lambda \mathrm{I}\right)^{-1} \mathrm{~A}^{\mathrm{T}}\left[\mathrm{Y}_{1}|| \mathrm{Y}_{\mathrm{N}_{\mathrm{Y}}}\right]
$$

which represents the estimate of functional observations, projected into the space of anatomically informed basis functions. The estimated signal in voxel-space is given by

$$
B_{\text {voxe }}^{\top}=A B^{\top} \text {. }
$$

Equivalently, let the columns of matrix $A_{\text {vertex }}$ be the folded basis functions in vertex-space $b_{G}^{j}$, then the estimated signal in vertex-space is given by

$$
B_{\text {vertex }}^{\top}=A_{\text {vertex }} B^{\top} \text {. }
$$

Inferences about evoked responses over time To make inferences about evoked responses, we have to characterize the parameter matrix B in the temporal domain. This could be done at three levels: (i) The first is in the space of the basis functions (Eq. (7)). (ii) The second is in the vertex-space (Eq. (9)), where we characterize the responses at each vertex in two dimensions and embed the results in three-dimensional space. (iii) Finally, we could project B back into voxel-space (Eq. (8)) and proceed with a conventional voxel-based analysis.

E ither a univariate or a multivariate technique could be used at all three levels. In this paper, we will illustrate a multivariate approach in basis function space. We use a combined approach of singular value decomposition (SVD), multivariate analysis of covariance (Mancova) and canonical variates analysis (CVA) as described in detail in (Friston et al., 1995) and (Chatfield and Collins, 1980).

As with a conventional analysis in voxel-space (e.g., as implemented in SPM99), we use various types of temporal basis functions or regressors: (i) condition encoding vectors, (ii) a constant, and (iii) a vector of global intracortical mean intensities. The condition encoding basis functions are the covariates of interest, the remainder represent covariates of no interest. Prior to fitting the model, we applied a highpass temporal filter with a cut-off frequency at 120 s/cycle.

Since we acquired the data using a rather long interscan time of $4 \mathrm{~s}$, we assumed that the highpass filter accounts for temporal auto-correlations, which are especially prominent at lower frequencies (Zarahn et al., 1997) and (Aguirre et al., 1997).

Let the temporal basis functions be $T=\left[T_{1} \mid T_{2}\right]$, where $T$ is a $\left(N_{Y} \times N_{T}\right)$-matrix, $N_{T}$ is the number of temporal basis functions, $\mathrm{T}_{1}$ and $\mathrm{T}_{2}$ are $\mathrm{N}_{\mathrm{Y}} \times \mathrm{N}_{\mathrm{T}_{1}}$ and $\mathrm{N}_{\mathrm{Y}} \times \mathrm{N}_{\mathrm{T}_{2}}$-matrices, $\mathrm{T}_{1}$ and $\mathrm{T}_{2}$ are orthogonal to each other, and $\mathrm{N}_{\mathrm{T}}=\mathrm{N}_{\mathrm{T}_{1}}+\mathrm{N}_{\mathrm{T}_{2}} . \mathrm{T}_{1}$ contains the covariates of interest and $T_{2}$ the covariates of no interest.

First we remove the effects of no interest by

$$
B_{C}=B-T_{2}\left(T_{2}^{T} T_{2}\right)^{-1} T_{2}^{T} B
$$

The SVD of the corrected parameter matrix $B_{C}$ is given by

$$
\mathrm{B}_{\mathrm{C}^{-}} \cup \vee \mathrm{W}^{\top}
$$

where $U$ and $W$ are orthogonal matrices and $V$ is a diagonal matrix. Let $\mathrm{B}_{\mathrm{P}}=\mathrm{U}_{\mathrm{J}} \mathrm{V}_{\mathrm{J}}$ a $\mathrm{N}_{\mathrm{Y}} \times \mathrm{N}_{\mathrm{J}}$ matrix, where the columns of $B_{p}$ contain the temporal expres- 


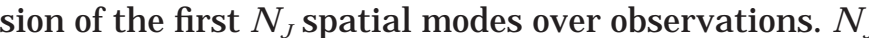
is found by thresholding the associated singular values contained in V as described in (Friston et al., 1995) and $\mathrm{U}_{\text {, }}$ and $\mathrm{V}_{\text {, }}$ are the accordingly reduced versions of $\mathrm{U}$ and $\mathrm{V}$.

Then the temporal model is given by

$$
\mathrm{B}_{\mathrm{P}}=\mathrm{T}_{1} \gamma+\epsilon_{\mathrm{T}}
$$

where $\gamma$ is a $N_{T_{1}} \times N_{J}$-matrix and $\epsilon_{T}$ (the residual errors) a $\mathrm{N}_{\mathrm{Y}} \times \mathrm{N}_{\mathrm{J}}$-matrix.

The least-squares estimate of $\gamma$ is

$$
\hat{\gamma}=\left(\mathrm{T}_{1}^{\mathrm{T}} \mathrm{T}_{1}\right)^{-1} \mathrm{~T}_{1}^{\mathrm{T}} \mathrm{B}_{\mathrm{P}} .
$$

Then the sums of squares and products due to error are

$$
\mathrm{R}_{\mathrm{E}}=\left(\mathrm{B}_{\mathrm{P}}-\mathrm{T}_{1} \hat{\gamma}\right)^{\top}\left(\mathrm{B}_{\mathrm{P}}-\mathrm{T}_{1} \hat{\gamma}\right)
$$

The sum of squares and products due to the effects of interest are

$$
H=\left(T_{1} \hat{\gamma}\right)^{\top}\left(T_{1} \hat{\gamma}\right)
$$

and the sum of squares and products under the null hypothesis (that the effects due to $T_{1}$ do not exist) are

$$
\mathrm{R}_{0}=\mathrm{B}_{\mathrm{P}}^{\mathrm{T}} \mathrm{B}_{\mathrm{P}}
$$

The significance of the effects of interest can be tested with Wilk's Lambda

$$
\Lambda=\frac{\left|R_{E}\right|}{\left|R_{0}\right|}
$$

as described in (Chatfield and Collins, 1980).

The characterization of the significant effects employs CVA, i.e., we find a matrix of canonical images $C$ $=\left[\mathbf{c}_{1}, \ldots, \mathbf{c}_{j}\right]$ such that the variance ratio $\left(\mathbf{c}_{\mathrm{m}}^{\top} \mathrm{H} \mathbf{c}_{\mathrm{m}}\right) /$ $\left(\mathbf{C}_{m}^{\top} R_{E} \mathbf{C}_{m}\right)$ is maximized successively for $m=1, \ldots, J$ under the condition that $\operatorname{cov}\left(\mathbf{c}_{m}, \mathbf{c}_{n}\right)=0$ for any $m, n$ with $1 \leq m, n \leq J$ and $m \neq n$. As it is shown below, the significant canonical modes can be visualized in the native voxel-space (functional or structural) or in vertex-space (folded, inflated, and flattened surfaces).

\section{VALIDATION STUDIES}

\section{Empirical Data}

Functional data and coregistration.

The functional data (EPI, gradient echo) were acquired on a clinical 1.5 T Siemens Vision unit (Siemens
GmbH, E rlangen) with TR $=168 \mathrm{~ms}$, TE $=79 \mathrm{~ms}$, flip angle $=90^{\circ}$. Each image consisted of $128 \times 128$ voxels, 20 partitions, transverse orientation, voxel size $1.8 \times$ $1.8 \times 3 \mathrm{~mm}^{3}$. F or each of the five subjects, the position and orientation of the slices were chosen to cover all the brain volume superior to the corpus callosum, in particular the primary sensorimotor hand area.

We acquired the T1-weighted structural and the E PI data in two successive measurements, i.e., the structural and the first scan of the functional data were al ready roughly in register. Subjects were instructed to keep still during the interval between sequences. To correct for small movements, the first functional scan was manually coregistered to the corresponding structural scan. Using SPM99 the functional scans were aligned to the first such that all measurements were in the same space.

\section{Simulated Data}

This section describes the simulated $\mathrm{FMRI}$ data used to validate AIBF and compare it with a voxel-based technique (i.e., SPM99).

All simulated data were generated from a single $\mathrm{fMRI}$ data set consisting of 93 volumes, acquired from one of the five subjects above using the same EPI gradient echo sequence. The only difference was that there was no activation condition; i.e., all scans were acquired under a continuous rest condition. As expected, this null data set showed no significant activations when tested with SPM 99 (maximum t score 4.39, corresponding to a $\mathrm{P}$ value of 0.93 , corrected for multiple comparisons). In particular, there were no activations in the region of the left primary sensorimotor cortex.

The next step was the addition of some well-defined simulated activations. We used the left hemisphere of the reconstructed cortical surface $S_{F}$ of subject and condition encoding temporal basis functions of the finger-tapping study to define the activation signal. We first chose a subset of vertices of $V_{F}$ (e.g., a circle of 3 $\mathrm{mm}$ diameter on the surface $\mathrm{S}_{\mathrm{F}}$ ) and defined these as the source of a BOLD effect. Then, we specified a (signal) time series in each vertex consisting of the condition encoding basis function. Next, we embedded the signal into voxel-space as described above. The voxelbased signal time-series was scaled such that the mean intensity difference between conditions was $5 \%$ of the mean (intracortical) global signal of the null data. Finally, we added the simulated signal in voxel-space to null data.

We generated three different sets of simulated data. These sets differed in their spatial configuration, i.e., in each set, a specific underlying cortical pattern of activation was simulated:

Singlesource. In this simulated data, we defined a single circular patch of 3-mm diameter on the gray matter surface as the source of an experimentally in- 
5

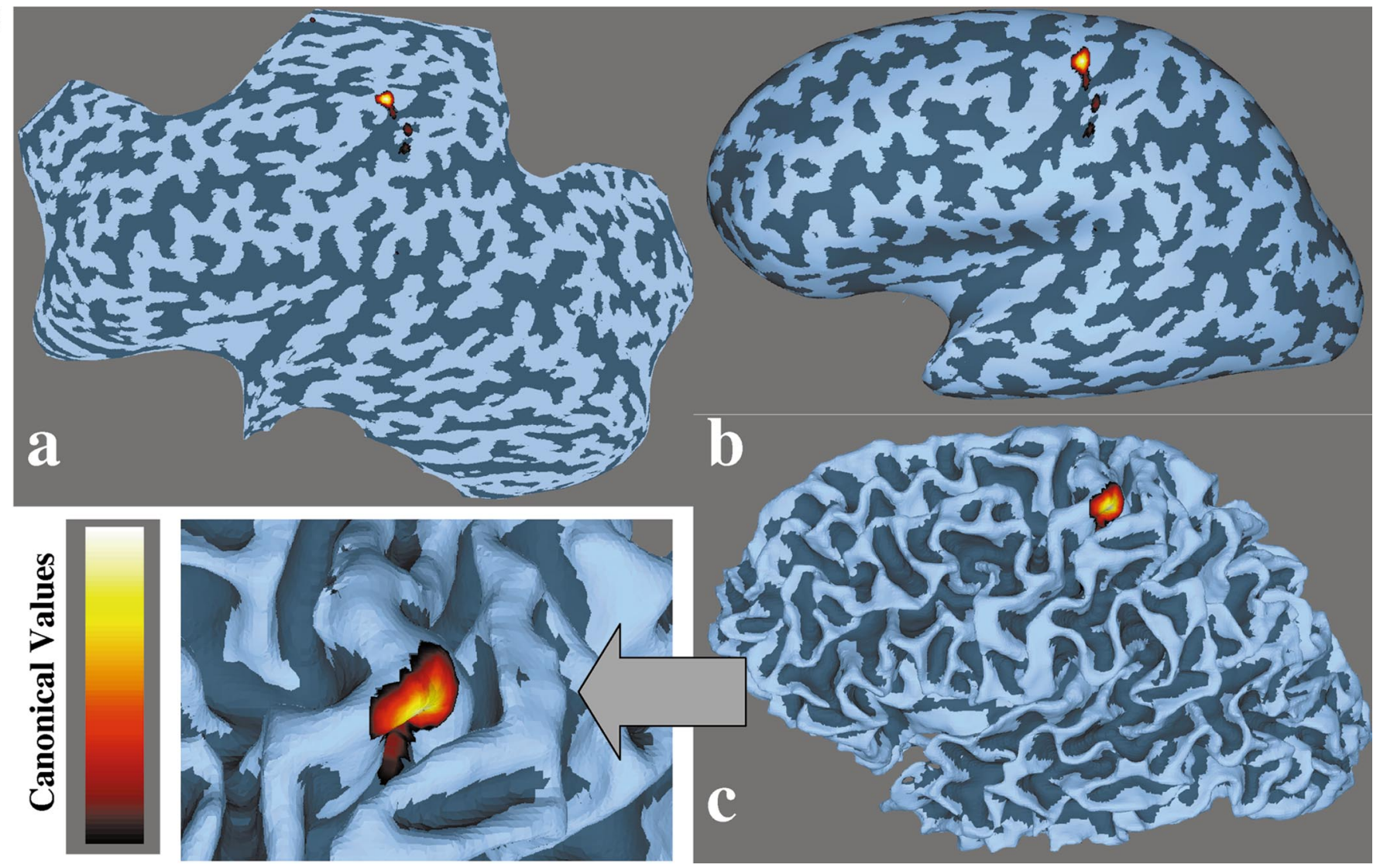

6
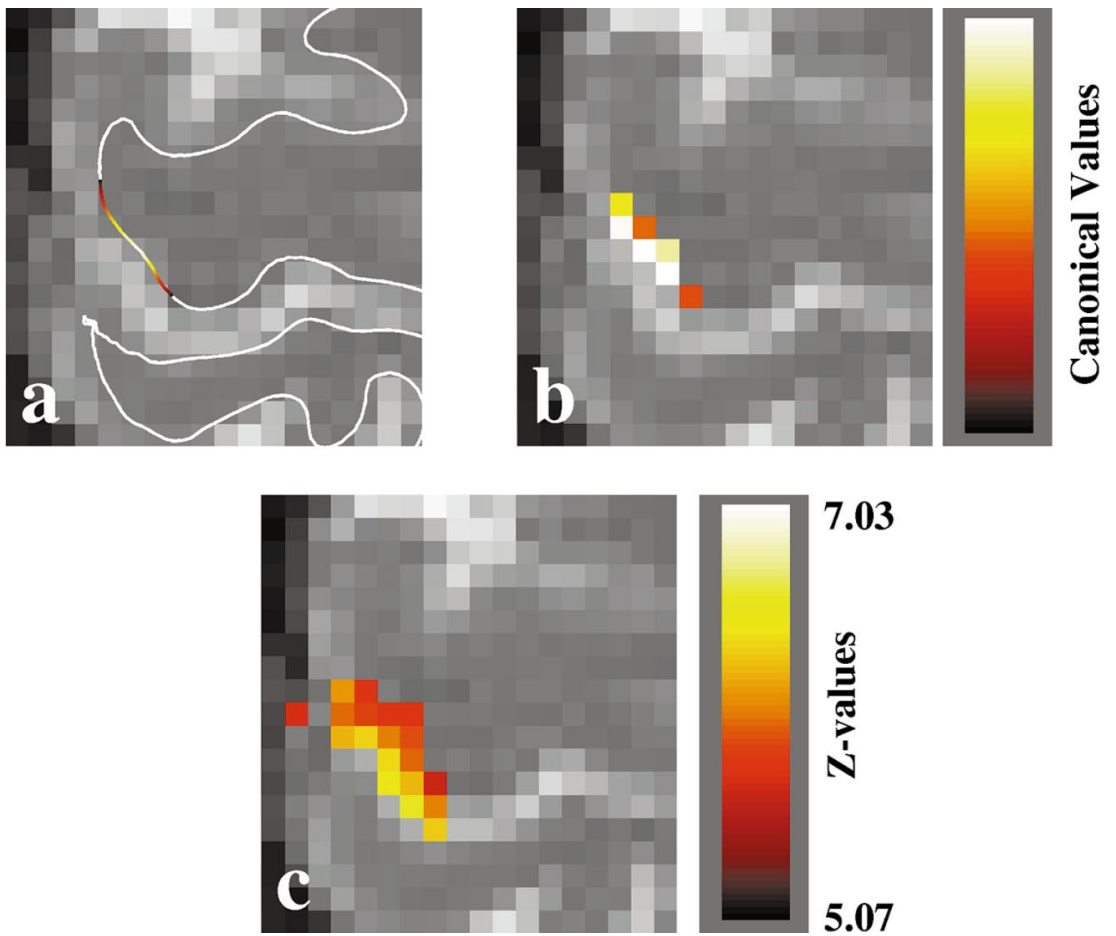

7.03

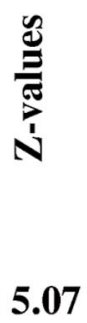

FIG. 5. AIBF results of one subject (left hemisphere) displayed on (a) the cortical flat map, (b) the inflated cortex, and (c) the folded gray matter surface.

FIG. 6. AIBF results in voxel-space (left hemisphere, central sulcus at level of presumed hand representation). Maximum of first canonical mode displayed on (a) the surface and (b) in voxel-space. In (c), the result of a SPM99-analysis for this slice is provided. 

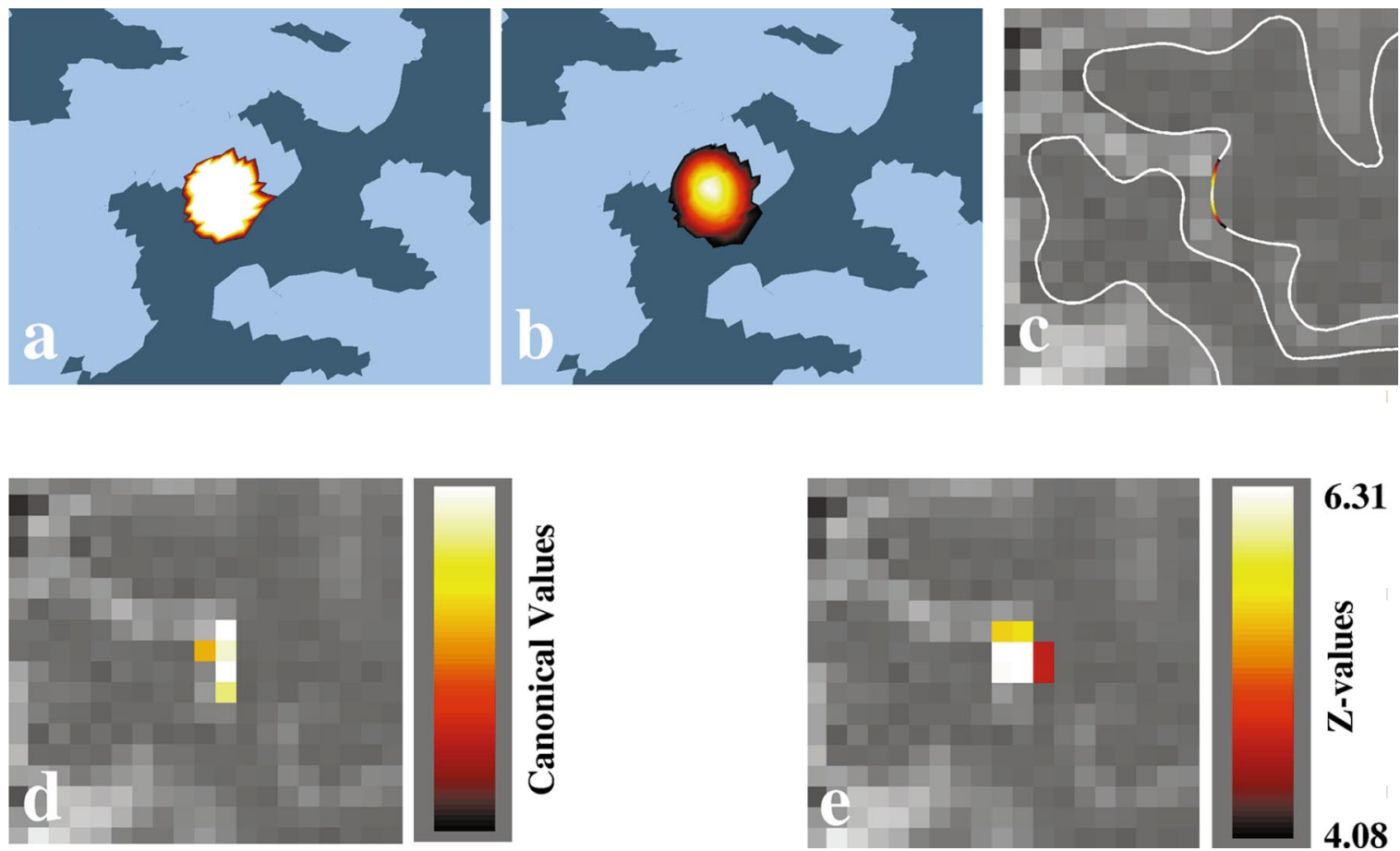

FIG. 7. Results on simulated data set "Single Source": (a) simulated configuration, (b) activation found by AIBF , (c) fit in vertex-space superimposed on transverse plane, (d) fit in voxel-space, (e) SPM99-result.

duced BOLD-effect. This patch was located on the anterior bank of the central sulcus roughly at the level of the presumed hand representation. In this analysis, the questions were, (i) whether the location and extent of the underlying BOLD-effect is precisely characterized by a linear combination of AIBF and, (ii) whether
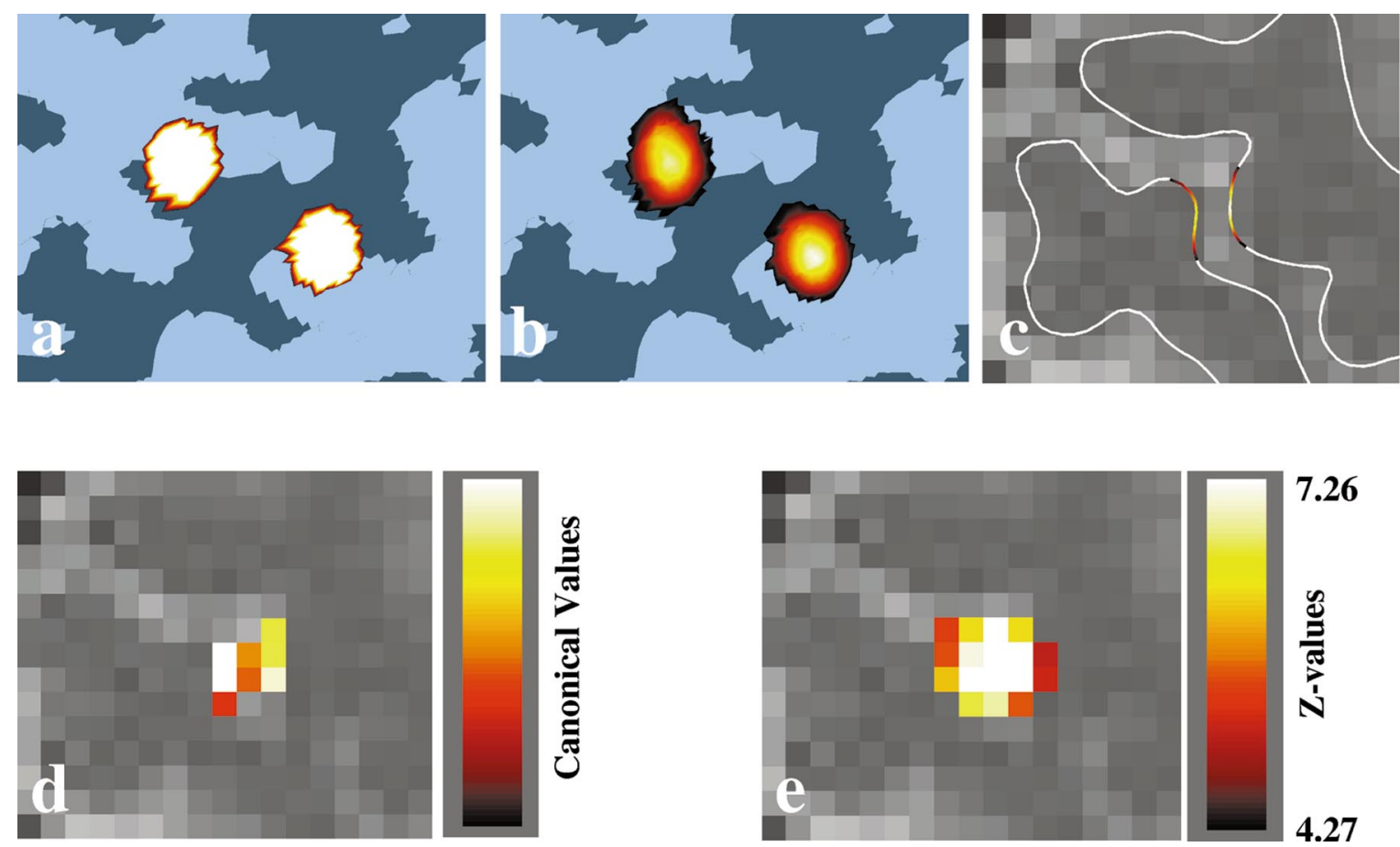

FIG. 8. Results on simulated data set "Two adjacent sources": (a) simulated configuration, (b) activation found by AIBF, (c) fit in vertex-space superimposed on transverse plane, (d) fit in voxel-space, (e) SPM99-result. 
any activation is spuriously mapped to the posterior bank of the sulcus.

Two adjacent sources. In this data set, we generated two sources of signal (circular patches of $3-\mathrm{mm}$ diameter), where one patch was located on the anterior bank of the central sulcus and the second was located on the opposite posterior bank, such that the Euclidean distance between the patches was minimal. The question here was whether AIBF separate the sources, which are close in Euclidean terms, but remote in terms of distance on the cortical surface.

Single source (extensively folded). Here, we generated a single circular patch of 5-mm diameter around the fundus of the central sulcus at the level of the presumed hand representation.

\section{Voxel-Based Analysis (SPM99)}

To characterize the differences between AIBF and a conventional voxel-based analysis, we analyzed all our data with SPM99. To do this, we smoothed the realigned data with a three-dimensional Gaussian lowpass-filter ([4 4 6] FWHM X-, y-, and z-direction). Statistical results were assessed using the general linear model and the theory of Gaussian random fields. All statistical results were thresholded at a significance level of $p<0.05$ (corrected for multiple comparisons).

\section{RESULTS}

\section{Fingertapping-Voxel-Based Analysis}

The results of a voxel-based analysis (SPM 99) of one subject are displayed in Fig. 4. As described in previous studies of motor tasks (Grafton et al., 1991; Wassermann et al., 1996; Puce et al., 1995; Schnitzler et al., 1997; Yousry et al., 1997), e.g., we found significant activation ( $P<0.05$ corrected) in the contralateral central sulcus (Fig. 4) and in the contralateral supplementory motor area. In two subjects, we observed significant $(P<0.05)$ ipsilateral activations in premotor and parietal areas. Due to the limited field of view, we did not assess effects in other locations, e.g., the cerebellum.

\section{Fingertapping-Anatomically I nformed}

Basis Functions

For each subject, we applied AIBF to the left hemispheres. We used basis functions with a distance of 2 $\mathrm{mm}$ between their centers and a width of $1 \mathrm{~mm}$, giving between 1242 and 2044 basis functions for each subject. In all five subjects, we identified a highly significant canonical mode within the left hemisphere $(\mathrm{P}<0.001)$.

To visualize the first canonical spatial mode in vertex-space, we took the linear combination of basis functions corresponding to this mode.
In Fig. 5, we display the thresholded mode of one subject on the individual folded, inflated, and flattened cortical surface. The arbitrary threshold $(60 \%$ of the maximum value) was used to enable the visualization of the highest canonical scores relative to the gyral pattern. We found, in each subject, a region of high canonical scores on the anterior bank of the central sulcus on the lateral side of the characteristic inverted Omega (Yousry et al., 1997). In all subjects except for one, this region also comprised the basis function with the maximal canonical score. In all subjects, there were also regions of high canonical scores on the posterior bank of the central sulcus close to those on the anterior bank.

To compare these findings to the results of a voxelbased analysis, we overlayed the two-dimensional color-coded gray matter surface on each transverse slice. In Fig. 6a the transverse slice containing the maximum of the canonical mode is displayed for one subject. Equivalently, we overlayed the thresholded first canonical mode defined in voxel-space (Eq. (3)) onto this slice (Fig. 6b). This encodes the same information as the surface representation, but shows the canonical mode in the space in which the regularized least-squares-fit to the functional observations was implemented. Finally, the result of the voxel-based analysis (SPM99) for this slice is shown in Fig. 6c.

\section{Simulated Data}

In all three simulated studies, we used a distance of $2 \mathrm{~mm}$ between centers of the basis functions and a width of $1 \mathrm{~mm}$ for each basis function.

Single source. The location of the simulated activation is shown in Fig. 7a. The result of using AIBF is shown in Figs. 7b-7d. The original location of the simulated activation was correctly identified on the cortical surface. The SPM99 result (Fig. 7e) is slightly blurred due to the smoothing, but the maximum peak of the cluster still correctly identifies the location.

Two adjacent sources. The locations of the two simulated activations is shown in Fig. 8a and the result of using AIBF in Figs. 8b-8d. Clearly, AIBF were able to identify and localize the two simulated activation sites, where AIBF seems to favor the source on the posterior bank. As shown in Fig. 8e, when employing a voxel-based method (SPM99) for the same data, we observe a contiguous cluster of activation, where the maximum peak is located between the two underlying sources.

Single source (extensively folded). The simulated activation was located in the fundus of the central sulcus and is shown in Fig. 9a. This location was correctly identified by AIBF (Figs. 9b-9d). However, the voxel-based analysis (Fig. 9e) localizes the maximum of the activation several $\mathrm{mm}$ lateral to the main source of activation. Again, this is a spurious effect due to the smoothing used prior to voxel-based analysis. 


\section{DISCUSSION}

In this work, we have proposed the use of anatomically informed basis functions (AIBF) for the analysis of functional MRI data. The method utilizes a new class of models, in which prior anatomical knowledge can be harnessed to better qualify the analysis in a neuroanatomical sense. We chose the reconstructed gray matter surface and the intrinsic smoothness of the BOLD effect over space as prior anatomical constraints in the examples presented in this paper. We applied AIBF to fMRI measurements of a motor task (fingertapping) in five subjects and compared the results with a voxel-based analysis (SPM 99). Additionally, we analyzed three sets of simulated data to characterize the salient differences between AIBF and voxel-based techniques.

In the following, we discuss the results as assessed by AIBF and SPM99. As an introductory remark, we note that a formal comparison between AIBF and SPM99 is a difficult task because of the differences in models and inference used in both methods. However, it is not our intention to show that AIBF or SPM is better or worse in some aspects than the other method. Our aim is to show that AIBF is a useful tool to look at functional data sets from a different and informed perspective.

There are three important differences between a voxel-based analysis and the class of AIBF presented.

\section{Three-Dimensional vs Two-Dimensional Smoothing}

When using AIBF, one does not apply any spatial lowpass filter to the data as is typical in a voxel-based analysis. In conventional analyses, a smoothing filter is used to enhance the sensitivity for a given signal width. The important point is that smoothing implicitly implements a model of the underlying data, namely one assumes that the underlying signal shape is independent of its location. Clearly, this model is not correct, since activations observed with fMRI usually follow the gyral pattern of gray matter such that there is a substantial interaction between position and the shape of the activation. The disadvantage of using a 3D-lowpass filter model is that activation effects at a given location are smeared into all adjacent locations, which can lead to physiologically implausible results (e.g., activations in white matter). F or the same reason, it is difficult to differentiate between activation sites that are close in Euclidean space, but remote from each other on the cortical surface (e.g., opposite banks of a sulcus). There is no doubt that one must spatially smooth functional observations to detect signal sources of a given width. However, the cortical sheet is highly convoluted and a voxel-based analysis of $\mathrm{fMRI}$ data based on exogenous smoothing is suboptimal in the sense that prior knowledge about structure-function relationships is not exploited. This renders it difficult to interpret statistical results in terms of the underlying physiology. AIBF, on the other hand, can be regarded as a spatially dependent smoothing filter, where the kernel is an explicit function of location. Intuitively, AIBF smoothes along the cortical surface such that any signal on the cortical surface, that conforms to the form of the basis functions, is detected with high sensitivity. In other words, AIBF intrinsically defines a space-varying smoothing kernel, which introduces only as much smoothness as is necessary for signal detection, while preserving functional topology.

\section{Superresolution}

AIBF introduces superresolution into the estimated solution, i.e., one can characterize the functional data on the high-resolution reconstructed surface. Since the gray matter surface can be approximated with subvoxel accuracy, we effectively exploit high-resolution knowledge in a low-resolution space by placing constraints on the solution. The benefit is that one can characterize the estimated solution not only in lowresolution (functional) voxel-space, but also, unambiguously, in the high-resolution vertex-space. The important point here is that the degree of superresolution depends primarily on the characteristics of the model specified, not on the data. Of course, in practice, the ultimate restriction is that the basis functions specified in vertex-space should lead to a sparse basis set in voxel-space, i.e., the lower the functional resolution, the less basis functions (as defined in Eq. (2)) are needed to span the measurement space in a sparse fashion.

\section{Effective Physiological Resolution}

By constraining the spatially dependent smoothing performed by AIBF to the gray matter surface, one implicitly suppresses signal in extraparenchymal space, e.g., from draining veins, which are located in CSF and can be several millimeters away from the original location of the neuronal activation. It is known that draining veins contribute to the BOLD-effects induced experimentally (Lai et al., 1993; Hoogenraad et al., 1999), particularly when using gradient-echo sequences as we did in our study. If one is interested in the temporal hemodynamic response of a given brain area, then the spatial differentiation between two underlying signal sources is not overly important. However, if one is interested in a spatial high-resolution characterization of activations, then the pattern of draining veins may limit the effective physiological resolution of gradient-echo fMRI.

\section{Surface Reconstruction}

The specification of a valid anatomical model is only possible in the context of AIBF, if (i) the reconstructed grey matter approximates the real cortical surface and 

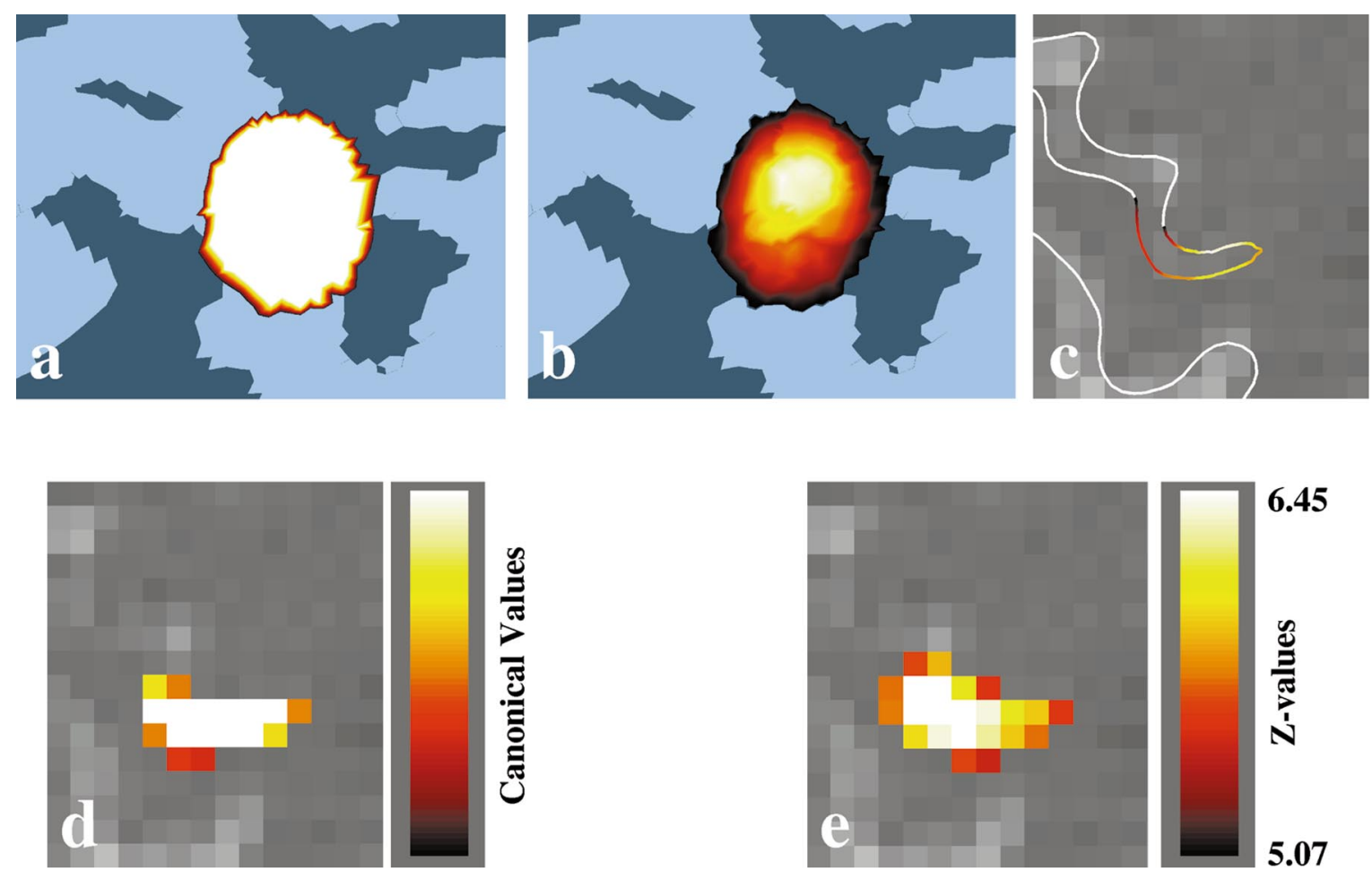

FIG. 9. Results on simulated data set "Single Source (extensively folded)": (a) simulated configuration, (b) activation found by AIBF, (c) fit in vertex-space superimposed on transverse plane, (d) fit in voxel-space, (e) SPM99-result.

(ii) the reconstructed grey matter surface also approximates the gray matter surface as measured in the low-resolution functional EPI-sequences. Visual inspection of the reconstructed surfaces overlayed on each individual's T1-weighted MRI showed that the first assumption was valid. The second assumption seems to be violated in the prefrontal region, where we observed some inconsistencies between functional and structural scans. However, for the primary sensorimotor cortical areas, we found that the second assumption was reasonable. A necessary prerequisite for this assumption is an accurate coregistration between the surface and functional observations.

\section{Distortions in Flat Maps}

The geometric distortions between the folded and flattened cortical surface, due to the curvature of the gray matter sheet, are unavoidable, especially when a whole hemisphere is transformed. Although cuts can diminish the ensuing distortions, they should be used sparsely to preserve the structure-function topology. Since our goal is to model a smooth distribution on the (folded) cortical surface (observed in voxel-space), the effect of geometric distortions is to render the local smoothness of the basis functions slightly variable and the ensuing change in fit (Eq. (7)) is minimal.

\section{Practical Issues}

Some practical issues must be considered when applying the method to functional data. In the current description, the Brainvoyager software was used to construct the anatomically informed model. However, the use of other surface reconstruction packages is possible at any stage of processing. We implemented our AIBF code in Matlab (Matlab 5.3, The Mathworks Inc.).

With regard to computer resources, the number of basis functions used to model the functional data is a limiting factor, since the parameter estimation (Eq. (4)) includes the matrix inversion of $\left(A^{\top} A+\gamma l\right)$. In our experience, the analysis of whole head volumes by AIBF is possible at a reasonable resolution (basis functions of 2-mm width) on a conventional workstation (256 MByte RAM, tested on a Sparc-Station and a Linux-PC). Note that the matrix inversion (Eq. (4)) need only to be done once, after this all functional volumes are fitted using the same projection matrix. The run-time of the whole process (generation of model, matrix inversion and fitting the data) is in the range of 5- $60 \mathrm{~min}$, again largely depending on the number of basis functions used.

\section{Generalization}

We want to identify an underlying spatiotemporal distribution of activity on a surface, where we only observe a low-resolution, noisy image of this distribution. Any valid prior spatial or spatiotemporal knowledge that can be used to constrain the solution will be usefully embodied in a set of AIBF ; e.g., one could try to 
model thick/thin stripes in the secondary visual area V2 directly on the cortical sheet.

AIBF can accomodate any form of prior anatomical knowledge given that an appropriate model can be represented as a set of basis functions. As in any inverse problem, the ensuing solution depends on the validity of the prior anatomical knowledge and the model specified. The generalization of the approach presented can be described in terms of potential adaptions.

The first obvious component is the definition of basis functions. Our choice of Gaussian basis functions was arbitrary. These can be replaced by any smooth, unimodal two-dimensional function, effectively implementing a lowpass filter kernel, e.g., sinc-functions. However, the change in the resulting fit is not as large as one might expect, because the transformation from high-resolution vertex- to low-resolution voxel-space effectively acts as an additional lowpass filter that renders different sets similar. A conceptually distinct kind of basis functions is a set of spatial cosine functions of different frequencies as used in stereotactic normalization (Ashburner and Friston, 1999).

In the case of other modalities like PET or SPECT (single photon emission computed tomography), one can model the intrinsic PSF by an additional convolution with a lowpass kernel $L$, which emulates the effects of underlying physical processes and its measurement. It is important to note that in this context the solution effectively implements a deconvolution of the original observations, since the estimated parameters can be used to project the observations in AIBF-space back into voxel-space without applying $L$.

We have used a zero-order regularization, which effectively weighs the solution in favor of small parameters. Although the regularization does not improve the overall fit, it stabilizes the fits of a specific basis function over time, since the associated parameter estimates are less susceptible to random errors of estimation. As a result, the contribution induced by the experimental paradigm is better reflected in the changes over time. Other regularization schemes can be used, e.g., first- or higher-order regularization as described by Press et al. (1992).

Finally, one could substitute the simple optimization method used to an iteratively adaptive scheme. An interesting extension would be to iteratively change the basis sets with respect to some goal function. Modifiable parameters could indude the widths of basis functions in $x$ and y-direction or positions on the cortical surface.

\section{CONCLUSION}

We have applied anatomically informed basis functions (AIBF) to a fMRI study and to simulated data.
We have shown that the method provides superresolution and higher sensitivity for signal sources within the gray matter sheet. In this work, we have used the location of the gray matter sheet as prior anatomical knowledge. More generally, AIBF can be used to incorporate any anatomical knowledge into functional analyses to constrain the resulting solution.

\section{ACKNOWLEDGMENT}

K.J .F. was supported by the Wellcome Trust.

\section{REFERENCES}

Aguirre, G., Zarahn, E., and D’Esposito, M. 1997. Empirical analyses of BOLD fMRI statistics. Neurol mage 5:199-212.

Ashburner, J ., and Friston, K. 1999. Nonlinear spatial normalization using basis functions. Hum. Brain Mapp. 7:254-266.

Chatfield, C., and Collins, A. 1980. Introduction to Multivariate Analysis. Chapman and Hall, London.

Friston, K. J ., Frith, C. D., Frackowiak, R. S. J ., and Turner, R. 1995. Characterizing dynamic brain responses with fMRI. Neurol mage 2:166-172.

Grafton, S. T., Woods, R. P., Mazziotta, J . C., and Phel ps, M. E. 1991. Somatotopic representation of the primary motor cortex in humans: Activation studies with cerebral blood flow and positron emission tomography. J . Neurophysiol. 66(3):735-743.

Hoogenraad, F., Hofman, M., Pouwels, P., Reichenbach, J ., Rombouts, S., and Haacke, E. 1999. Sub-millimeter fMRI at 1.5 tesla: Correlation of high resolution with low resolution measurements. J . Magn. Reson. Imag. 9:475- 482.

Lai, S., Hopkins, A., Haacke, E., Li, D., Wassermann, B., Buckley, P., Friedman, L., Meltzer, H., Hedera, P., and Friedland, R. 1993. Identification of vascular structures as a major source of signal contrast in high resolution $2 \mathrm{~d}$ and $3 \mathrm{~d}$, functional activation imaging of the motor cortex at 1.5t: Preliminary results. Magn. Reson. Med. 30:387-392.

Linden, D., Kallenbach, U., Heinecke, A., Singer, W., and Goebel, R. 1999. The myth of upright vision. a psychophysical and functional imaging study of adaptation to inverting spectacles. Perception in press.

Press, W. H., Teukolsky, S. A., and Vetterling, W. T. 1992. Numerical Recipes in C: TheArt of Scientific Computing. Cambridge Univ. Press.

Puce, A., Luby, R. C. M., McCarthy, G., Nobre, A., and Spencer, D. 1995. Functional magnetic resonance imaging of sensory and motor cortex: Comparison with electrophysiological localization. J . Neurosurg. 83:262-270.

Schnitzler, A., Salenius, S., Salmelin, R., J ousm"aki, V., and Hari, R. 1997. Involvement of primary motor cortex in motor imagery: A neuromagnetic study. Neuroimage 6:201-208.

Wassermann, E. M., Wang, B., Zeffiro, T. A., Sadato, N., PascualLeone, A., Toro, C., and Hallett, M. 1996. Locating the motor cortex on the MRI with transcranial magnetic stimulation and PET. Neuroimage 3:1-9.

Yousry, T., Schmid, U. D., Schmidt, H. A., Peraud, A., Buettner, A., and Winkler, P. 1997. Localization of the motor hand area to a knob on the precentral gyrus. Brain 120:141-157.

Zarahn, E., Aguirre, G., and D'Esposito, M. 1997. Empiral analyses of BOLD fMRI statistics. Neurol mage 5:179-197. 